\title{
Mikrobiologische Aspekte in der Trinkwasserversorgung
}

Trotz sehr hoher Qualitätsstandards und dem hervorragenden Ruf, den das österreichische Trinkwasser im In- und Ausland genießt, blicken die Wasserversorger einer Vielzahl an Herausforderungen entgegen. Die vermutlich größten dieser Herausforderungen, denen die Wasserwirtschaft in den kommenden Jahrzehnten begegnet, stellen die Folgen des Klimawandels dar.

Durch erhöhte Durchschnittstemperaturen im Jahresverlauf, aber auch durch Extremwetterlagen - wie z. B. länger anhaltende Hitzeperioden - ist mit einer Zunahme der Temperaturen bei den Wasserressourcen Grundwasser und Oberflächengewässer, aber auch mit einer Zunahme der Wassertemperatur entlang der Versorgungskette zu rechnen. Da die Umgebungstemperatur einen entscheidenden Faktor für das Wachstum und die Vermehrung von Mikroorganismen darstellt, kann man langfristig mit Veränderungen in der mikrobiologischen Zusammensetzung von Wasserproben rechnen.

Neben den direkten Auswirkungen auf die Wasserqualität ist durch den Klimawandel auch mit Veränderungen bzw. Umverteilungen im Wasserdargebot zu rechnen. Um eine weitgehende Versorgungssicherheit mit Trinkwasser zu gewährleisten, wird es für Wasserversorger laufend wichtiger, zusätzliche Ressourcen zu erschließen. In diesem Kontext werden Uferfiltrate aufgrund ihrer hohen Mengenverfügbarkeit und der zielgerichteten Entnahmemöglichkeit immer attraktiver. Entlang der österreichischen Flüsse wie Donau, Rhein und Mur werden seit Jahrzehnten Uferfiltrate für die Gewinnung von Trinkwasser genutzt.

Die hydrologischen und hydraulischen Randbedingungen der Fließgewässer beeinflussen maßgeblich die mikrobiologische Dynamik im Uferfiltrat. Hohe Durchlässigkeit im Untergrund, hohe Entnahmeraten und hohe Wasserspiegellagen ergeben einen gesteigerten Gradient der Infiltrationsströmung in Richtung Entnahmebrunnen und damit zu einer relativ kurzen Aufenthaltszeit im Untergrund. Diese Wechselwirkungen haben in weiterer Folge einen direkten Einfluss auf die mikrobielle Zusammensetzung des Wassers.

Methodische Entwicklungen in der mikrobiologischen Analyse von Wasserproben, wie beispielsweise Durchflusszytometrie und DNA-Sequenzierungsmethoden, erlauben einen weitaus umfassenderen Einblick in die mikrobiologische Beschaffenheit von Wasserproben. So kann durch den Einsatz von Methoden mit hohem Probendurchsatz - wie bei der Durchflusszytometrie - die räumliche und zeitliche Auflösung der untersuchten Proben stark gesteigert werden. DNA-Sequenzierungsmethoden erlauben darüber hinaus einen tiefgehenden und detaillierten Einblick in die mikrobielle Zusammensetzung, insbesondere in Bezug auf sonst nicht kultivierbare Organismen, und schaffen dadurch einen weit umfassenderen Einblick in diese Lebensgemeinschaften.

Diesen Veränderungen der Randbedingungen steht die Erwartung der Konsumenten an eine sehr hohe Qualität und Versorgungssicherheit der Trinkwasserversorgung, trotz sich gegebenenfalls ungünstig verändernder Randbedingungen gegenüber. Diese Anforderungen bewirken in der Folge Anpassungen in den gesetzlichen Grundlagen. Das wird aktuell bei der noch nicht abgeschlossenen Überarbeitung der EU-Trinkwasserrichtlinie sichtbar, die versucht, entsprechend dem aktuellen Stand des Wissens die Sicherheit und Qualität der Trinkwasserversorgung auch in Zukunft zu gewährleisten. 
Univ.-Prof. DI Dr. T. Ertl (西) •

PD DI Dr. R. Perfler (价

Department Wasser, Atmosphäre,

Umwelt, Institut für Siedlungswas-

serbau, Industriewasserwirtschaft

und Gewässerschutz, Universität für

Bodenkultur Wien,

Muthgasse 18,

1190 Wien, Österreich

thomas.ert|@boku.ac.at; rein-

hard.perfler@boku.ac.at 